Draft Version September 5, 2018

Preprint typeset using $\mathrm{L}^{A} \mathrm{~T}_{\mathrm{E}} \mathrm{X}$ style emulateapj v. 11/10/09

\title{
FORMING PLANETESIMALS BY GRAVITATIONAL INSTABILITY: I. THE ROLE OF THE RICHARDSON NUMBER IN TRIGGERING THE KELVIN-HELMHOLTZ INSTABILITY
}

\author{
Aaron T. LeE $^{1}$, Eugene Chiang ${ }^{1,2}$, Xylar Asay-Davis ${ }^{3}$, And Joseph Barranco ${ }^{4}$ \\ Draft version September 5, 2018
}

\begin{abstract}
Gravitational instability (GI) of a dust-rich layer at the midplane of a gaseous circumstellar disk is one proposed mechanism to form planetesimals, the building blocks of rocky planets and gas giant cores. Self-gravity competes against the Kelvin-Helmholtz instability (KHI): gradients in dust content drive a vertical shear which risks overturning the dusty subdisk and forestalling GI. To understand the conditions under which the disk can resist the KHI, we perform three-dimensional simulations of stratified subdisks in the limit that dust particles are small and aerodynamically well coupled to gas, thereby screening out the streaming instability and isolating the KHI. Each subdisk is assumed to have a vertical density profile given by a spatially constant Richardson number $R i$. We vary $R i$ and the midplane dust-to-gas ratio $\mu_{0}$ and find that the critical Richardson number dividing KHunstable from $\mathrm{KH}$-stable flows is not unique; rather $R i_{\text {crit }}$ grows nearly linearly with $\mu_{0}$ for $\mu_{0}=$ 0.3-10. Plausibly a linear dependence arises for $\mu_{0} \ll 1$ because in this regime the radial Kepler shear replaces vertical buoyancy as the dominant stabilizing influence. Why this dependence should persist at $\mu_{0}>1$ is a new puzzle. The bulk (height-integrated) metallicity is uniquely determined by $R i$ and $\mu_{0}$. Only for disks of bulk solar metallicity is $R i_{\text {crit }} \approx 0.2$, close to the classical value. Our empirical stability boundary is such that a dusty sublayer can gravitationally fragment and presumably spawn planetesimals if embedded within a solar metallicity gas disk $\sim 4 \times$ more massive than the minimum-mass solar nebula; or a minimum-mass disk having $\sim 3 \times$ solar metallicity; or some intermediate combination of these two possibilities. Gravitational instability seems possible without resorting to the streaming instability or to turbulent concentration of particles.

Subject headings: hydrodynamics — instabilities — planetary systems: protoplanetary disks — planets and satellites: formation
\end{abstract}

\section{INTRODUCTION}

In the most venerable scenario for forming planetesimals, dust particles in circumstellar gas disks are imagined to settle vertically into thin sublayers ("subdisks") sufficiently dense to undergo gravitational instability (Safronov 1969; Goldreich \& Ward 1973; for a review of this and other ways in which planetesimals may form, see Chiang \& Youdin 2010, hereafter CY10). Along with this longstanding hope comes a longstanding fear that dust remains lofted up by turbulence. Even if we suppose that certain regions of the disk are devoid of magnetized turbulence because they are too poorly ionized to sustain magnetic activity Gammie 1996; Bai \& Goodman 2009), the dusty sublayer is susceptible to a Kelvin-Helmholtz shearing instability (KHI; Weidenschilling 1980) 5

\footnotetext{
a.t.lee@berkeley.edu

${ }^{i}$ Department of Astronomy, University of California, Berkeley, CA 94720

2 Department of Earth and Planetary Science, University of California, Berkeley, CA 94720

${ }^{3}$ Center for Nonlinear Studies, Los Alamos National Laboratory, Los Alamos, NM 87545

4 Department of Physics and Astronomy, San Francisco State University, San Francisco, CA 94132

5 Goldreich \& Ward (1973) also recognized that the sublayer would be shear-unstable, but unlike Weidenschilling (1980), overlooked the possibility that the KHI may forestall gravitational instability.
}

\subsection{Basic Estimates}

The KHI arises because dust-rich gas at the midplane rotates at a different speed from dust-poor gas at altitude. The background radial pressure gradient $\partial P / \partial r$ causes dust-free gas at disk radius $r$ to rotate at the slightly non-Keplerian rate

$$
\Omega_{\mathrm{F}}=\Omega_{\mathrm{K}}(1-\eta)
$$

where $\Omega_{\mathrm{K}}$ is the Kepler angular frequency,

$$
\eta=\frac{-\left(1 / \rho_{\mathrm{g}}\right) \partial P / \partial r}{2 \Omega_{\mathrm{K}}^{2} r} \approx 8 \times 10^{-4}\left(\frac{r}{\mathrm{AU}}\right)^{4 / 7}
$$

is a dimensionless measure of centrifugal support by pressure, and $\rho_{\mathrm{g}}$ is the density of gas (e.g., Nakagawa et al. 1986; Cuzzi et al. 1993). The numerical evaluation is based on the minimum-mass solar nebula derived by CY10. Unlike dust-free gas, dust-rich gas is loaded by the extra inertia of solids and must rotate at more nearly the full Keplerian rate to remain in centrifugal balance. Variations in the dust-to-gas ratio $\rho_{\mathrm{d}} / \rho_{\mathrm{g}}$ with height $z$ result in a vertical shear $\partial v_{\phi} / \partial z$ from which free energy is available to overturn the dust layer.

The shearing rate across a layer of thickness $\Delta z$ is given to order of magnitude by

$$
\begin{aligned}
\left|\frac{\partial v_{\phi}}{\partial z}\right| \sim \frac{\Delta v_{\phi}}{\Delta z} & =\frac{1}{\Delta z} \frac{\mu_{0}}{1+\mu_{0}} \eta \Omega_{\mathrm{K}} r \\
& \approx \frac{25}{\Delta z} \frac{\mu_{0}}{1+\mu_{0}}\left(\frac{r}{\mathrm{AU}}\right)^{1 / 14} \mathrm{~m} \mathrm{~s}^{-1}
\end{aligned}
$$


where $\rho_{\mathrm{d}} / \rho_{\mathrm{g}}=\mu_{0}$ at the midplane and $\rho_{\mathrm{d}} / \rho_{\mathrm{g}} \ll 1$ at altitude (for more details see CY10, or $\$ \$ 2.122 .2$ of this paper). For $\mu_{0} \gg 1$ the velocity difference $\Delta v_{\phi}$ saturates at a speed $\eta \Omega_{\mathrm{K}} r \sim 25(r / \mathrm{AU})^{1 / 14} \mathrm{~m} \mathrm{~s}^{-1}$, well below the gas sound speed $c_{\mathrm{S}} \sim 1 \mathrm{kms}^{-1}$. That the flow is highly subsonic motivates what simulation methods we employ in our study.

We might expect the flow to be stabilized if the BruntVäisälä frequency

$$
\begin{aligned}
\omega_{\text {Brunt }} & =\left(\frac{-g}{\rho} \frac{\partial \rho}{\partial z}\right)^{1 / 2} \\
& \sim\left(\frac{\mu_{0}}{1+\mu_{0}}\right)^{1 / 2} \Omega_{\mathrm{K}}
\end{aligned}
$$

of buoyant vertical oscillations is much larger than the vertical shearing rate. For the order-of-magnitude evaluation in (4) we approximate the vertical gravitational acceleration $g$ as the vertical component of stellar gravity $-\Omega_{\mathrm{K}}^{2} \Delta z$ (no self-gravity), and the density gradient $\rho^{-1} \partial \rho / \partial z \sim\left(\rho_{\mathrm{d}}+\rho_{\mathrm{g}}\right)^{-1} \Delta\left(\rho_{\mathrm{d}}+\rho_{\mathrm{g}}\right) / \Delta z \sim\left(\rho_{\mathrm{d}}+\right.$ $\left.\rho_{\mathrm{g}}\right)^{-1} \Delta \rho_{\mathrm{d}} / \Delta z$. The last approximation relies in part on the dust density $\rho_{\mathrm{d}}$ changing over a lengthscale $\Delta z$ much shorter than the gas scale height. Both $\left|\partial v_{\phi} / \partial z\right|$ and $\omega_{\text {Brunt }}$ shrink as $\mu_{0}$ decreases 6

For two-dimensional, heterogeneous, unmagnetized flow, a necessary but not sufficient condition for instability is given by the Richardson number:

$R i \equiv \frac{-(g / \rho)(d \rho / d z)}{\left(d v_{\phi} / d z\right)^{2}}<1 / 4$ is necessary for instability

(Miles 1961; see the textbook by Drazin \& Reid 2004). The Richardson number is simply the square of the ratio of the stabilizing Brunt frequency (4) to the destabilizing vertical shearing frequency (3). The critical value of $1 / 4$ arises formally but can also be derived heuristically by energy arguments (e.g., Chandrasekhar 1981). The Richardson criterion does not formally apply to our dusty subdisk, which represents a three-dimensional flow: the KHI couples vertical motions to azimuthal motions, while the Coriolis force couples azimuthal motions to radial motions. (For how the Richardson criterion may not apply to magnetized flows, see Lecoanet et al. 2010.) Nevertheless we may hope the Richardson number is useful as a guide, as previous works have assumed (Sekiva 1998; Youdin \& Shu 2002; Youdin \& Chiang 2004).

In this spirit let us use the Richardson criterion to estimate the thickness of a marginally $\mathrm{KH}$-unstable dust

\footnotetext{
6 But not indefinitely. In the limit $\mu_{0} \rightarrow 0$, the vertical shearing and Brunt frequencies reach minima set by pressure and temperature gradients in gas (see, e.g., Knobloch \& Spruit 1985). The limit $\mu_{0} \rightarrow 0$ is not relevant for our study and not captured by either (3) or (4).
}

layer. Substitution of (3) and (44) into (5) reveals that7

$$
\Delta z \approx\left(\frac{\mu_{0}}{1+\mu_{0}}\right)^{1 / 2} R i^{1 / 2} \eta r .
$$

Since the gas scale height $H_{\mathrm{g}}=c_{\mathrm{s}} / \Omega_{\mathrm{K}}$ and $\eta \sim\left(H_{\mathrm{g}} / r\right)^{2}$, equation (6) indicates that for $\mu_{0}>1$ the marginally unstable dust sublayer is $\sim R i^{1 / 2} H_{\mathrm{g}} / r \sim 0.02 R i^{1 / 2}$ times as thin as the gas disk in which it is immersed. Those KH-unstable modes that disrupt the layer should have azimuthal wavelengths - and by extension radial wavelengths, because the Kepler shear turns azimuthal modes into radial ones - that are comparable to $\Delta z$. Shorter wavelength modes cannot overturn the layer, while longer wavelength modes grow too slowly Gómez \& Ostriker 2005).

How does disk rotation affect the development of the KHI? In a linear analysis, Ishitsu \& Sekiva (2003) highlight the role played by the Keplerian shear, characterized by the strain rate

$$
\left|\frac{\partial \Omega_{\mathrm{K}}}{\partial \ln r}\right|=\frac{3 \Omega_{\mathrm{K}}}{2}
$$

in limiting the growth of $\mathrm{KH}$-unstable modes. The radial shear is implicated because azimuthal motions excited by the KHI are converted to radial motions by the Coriolis force; moreover, the non-axisymmetric pattern excited by the KHI is wound up, i.e., stretched azimuthally by the radial shear. The Kepler rate $\left|\partial \Omega_{K} / \partial \ln r\right|$ is at least as large as $\omega_{\text {Brunt }}$, and can dominate the latter when $\mu_{0}$ is small. This suggests that $R i$ does not capture all the relevant dynamics - a concern already clear on formal grounds. In this paper we address this concern head-on, using fully 3D numerical simulations to assess the role of the Richardson number in governing the stability of the dust layer.

\subsection{Our Study in Relation to Previous Numerical Simulations}

Three-dimensional shearing box simulations of the KHI in dusty subdisks, performed in the limits that dust is perfectly coupled to gas and disk self-gravity is negligible, demonstrate the importance of the Kepler shear. Compared to rigidly rotating disks Gómez \& Ostriker 2005; Johansen et al. 2006), radially shearing disks are far more stable (Chiang 2008; Barranco 2009). The relevance of $R i$, or lack thereof, may be assessed by simulating flows with initially spatially constant $R i$ (Sekiva 1998; Youdin \& Shu 2002), and varying $R i$ from run to run to see whether dust layers turn over. Chiang (2008, hereafter C08) found that when $\mu_{0}>1$, dust layers for which $R i<0.1$ overturn, while those for which $R i>0.1$ do not. In retrospect, we might have anticipated this result, that the critical value $R i_{\text {crit }}$ dividing stable from unstable runs lies near the canonical value of $1 / 4$, at least

\footnotetext{
7 This order-of-magnitude expression for the dust layer thickness, and the related equation (3) which approximates the vertical shear, are each smaller than their counterparts given by Youdin \& Shu (2002, page 499, first full paragraph) by a factor of $(1+\mu)$. This is because Youdin \& Shu (2002) evaluate quantities deep inside the layer, within a density cusp at the midplane, whereas we are interested in quantities averaged across the entire layer. The difference does not change either our conclusions or theirs.
} 
for $\mu_{0}>1$, because in this regime of parameter space all the frequencies of the problem are comparable to each other: $\left|\partial v_{\phi} / \partial z\right| \sim \omega_{\text {Brunt }} \sim\left|\partial \Omega_{\mathrm{K}} / \partial \ln r\right| \sim \Omega_{\mathrm{K}}$ when $\mu_{0}>1$ and $R i \approx 0.1-1$. But other simulations of $\mathrm{C} 08$ also make clear that $R i$ does not alone determine stability under all circumstances. For $\mu_{0} \approx 0.2-0.4, R i_{\text {crit }}$ was discovered to drop substantially to $\sim 0.02$ (see his runs S9S12). Chiang (2008) speculated that the baroclinic nature of the flow may be responsible (Knobloch \& Spruit 1985, 1986), but no details were given.

In addition to being left unexplained, the findings of $\mathrm{C} 08$ require verification. Parameter space was too sparsely sampled to discern trends with confidence. Concerns about numerics - e.g., biases introduced by box sizes that were too small, resolutions too coarse, and runs terminated too early - also linger. At least one numerical artifact marred the simulations of C08: the KHI manifested first at the "co-rotation" radius where the mean azimuthal flow speed was zero (see his figure 8). But in a shearing box, by Galilean invariance, there should be no special radius. It was suspected, but not confirmed, that errors of interpolation associated with the grid-based advection scheme used by C08 artificially suppressed the KHI away from co-rotation.

For the problem at hand, the spectral code developed by Barranco \& Marcus (2006) and modified by Barranco (2009, hereafter B09) to treat mixtures of dust and gas is a superior tool to the grid-based ZEUS code utilized by C08. Working in Fourier space rather than configuration space, the simulations of the KHI by B09 did not betray the co-rotation artifact mentioned above. Spectral methods, often used to model local (WKB) dynamics, are appropriate here because the structures of interest in the subdisk have dimensions tiny compared to the disk radius (by at least a factor $R i^{1 / 2} \eta$ according to equation [6) and even the gas scale height. At the same computational expense, spectral algorithms typically achieve greater effective spatial resolution than their grid-based counterparts (Barranco \& Marcus 2006). Another advantage enjoyed by the B09 code is that it employs the anelastic approximation, which is designed to treat subsonic flows such as ours. Having filtered away sound waves, anelastic codes are free to take timesteps set by how long it takes fluid to advect across a grid cell (which themselves move at the local orbital velocity in a shearing coordinate system). By contrast, codes such as ZEUS take mincing steps limited by the time for sound waves to cross a grid cell. The latter constraint is the usual Courant condition for numerically solving problems in compressible fluid dynamics. It was unnecessarily applied by C08 to a practically incompressible flow.

In this paper we bring all the advantages of the spectral, anelastic, shearing box code of B09 to bear on the problems originally addressed by C08. We assess numerically the stability of flows characterized by constant Richardson number $R i$, systematically mapping out the stability boundary in the parameter space of $R i$, midplane dust-to-gas ratio $\mu_{0}$, and bulk metallicity $\Sigma_{\mathrm{d}} / \Sigma_{\mathrm{g}}$ (the height-integrated surface density ratio of dust to gas). Though our simulations may still be underresolved, we rule out box size as a major influence on our results. We offer some new insight into why $R i$ is not a sufficient predictor of stability. And in the restricted context of our constant $R i$ flows, we assess the conditions necessary for the midplane to become dense enough to trigger gravitational instability on a dynamical time.

\subsection{The Perfect Coupling Approximation vs. The Streaming Instability vs. Turbulent Concentration Between Eddies}

Following C08 and B09, we continue to work in the limit that dust is perfectly coupled to gas, i.e., in the limit that particles are small enough that their frictional stopping times $t_{\text {stop }}$ in gas can be neglected in comparison to the dynamical time $\Omega_{\mathrm{K}}^{-1}$. The perfect coupling approximation allows us to screen out the streaming instability which relies on a finite stopping time and which is most powerful when particles are marginally coupled, i.e., when $\tau_{\mathrm{s}} \equiv \Omega_{\mathrm{K}} t_{\text {stop }} \sim 0.1-1$ (Youdin \& Goodman 2005). Numerical simulations have shown that when an order-unity fraction of the disk's solids is in particles having $\tau_{\mathrm{s}}=0.1-1$, the streaming instability clumps them strongly and paves the way for gravitational instability (e.g., Johansen et al. 2007, 2009). The particle sizes corresponding to $\tau_{\mathrm{s}}=1$ depend on the properties of the background gas disk, as well as on the particle's shape and internal density; under typical assumptions, marginally coupled particles are decimeter to meter-sized.

It remains debatable whether a substantial fraction of a disk's solid mass is in marginally coupled particles at the time of planetesimal formation, as current proposals relying on the streaming instability assume. Particle size and shape distributions are not well constrained by observations (though see, e.g., Wilner et al. 2005, who showed that centimeter-wavelength fluxes from a few $\mathrm{T}$ Tauri stars are consistent with having been emitted by predominantly centimeter-sized particles). Measuring $\tau_{\mathrm{s}}$ in disks also requires knowing the gas density, but direct measurements of the gas density at disk midplanes do not exist. Marginally coupled particles - sometimes referred to as "meter-sized boulders" - also face the longstanding problem that they drift onto the central star too quickly, within hundreds of years from distances of a few AU in a minimum-mass disk. Johansen et al. (2007) claimed to solve this problem by agglomerating all the boulders into Ceres-mass planetesimals via the streaming instability before they drifted inward. Their simulation presumed, however, that all of the disk's solids began boulder-sized. The concern we have is that even if particle-particle sticking could grow boulders (and sticking is expected to stall at centimeter sizes; Blum \& Wurm 2008; CY10), the disk's solids may not be transformed into boulders all at once. Rather, marginally coupled bodies may initially comprise a minority population on the extreme tail of the particle size distribution. Unless they can transform themselves from a minority to a majority within the radial drift timescale, they would be lost from the nebula by aerodynamic drag.

By focussing on the dynamics of the smallest, most well entrained particles having $\tau_{\mathrm{s}} \ll 1$, our work complements that which relies on the streaming instability. We would argue further that the well coupled limit is potentially more relevant for planet formation. If even the smallest particles having sizes $\ll \mathrm{cm}$ can undergo gravitational collapse to form kilometer-sized or larger planetes- 
imals, nature will have leapfrogged over the marginally coupled regime, bypassing the complications and uncertainties described above.

Particle clumping is not restricted to marginally coupled particles via the streaming instability. Small $\tau_{\mathrm{s}}$ particles also clump within the interstices of turbulent, high vorticity eddies (Maxev 1987; Eaton \& Fessler 1994; Cuzzi et al. 2008, and references therein; for a review, see CY10). This particle concentration mechanism presumes some gas turbulence, which may be present in the marginally $\mathrm{KH}$-unstable state to which dust settles. Our simulations cannot capture this phenomenon. However, on the scales of interest to us, turbulent clumping might only be of minor significance. Particles of given $t_{\text {stop }}$ are concentrated preferentially by eddies that turn over on the same timescale. Thus the degree of concentration depends sensitively on particle size and the turbulent spectrum. At least in Kolmogorov turbulence, the smallest eddies concentrate particles most strongly because they have the greatest vorticity. The smallest eddies at the inner scale of Kolmogorov turbulence have sizes $\ell_{\mathrm{i}} \sim \nu^{3 / 4} t_{\mathrm{o}}^{1 / 4} / \delta v_{\mathrm{o}}^{1 / 2}$, where $\nu$ is the molecular kinematic viscosity, and $t_{\mathrm{o}}$ and $\delta v_{\mathrm{o}}$ are the turnover time and velocity of the largest, outer scale eddy. Given $\delta v_{\mathrm{o}} \sim \eta \Omega_{\mathrm{K}} r \sim 25(r / \mathrm{AU})^{1 / 14} \mathrm{~m} / \mathrm{s}, t_{\mathrm{o}} \sim \Omega_{\mathrm{K}}^{-1}$, and values of $\nu$ based on the nebular model of CY10, we estimate that $\ell_{\mathrm{i}} \sim 10^{3}(r / \mathrm{AU})^{127 / 56} \mathrm{~cm}$. This is far smaller than the sublayer thicknesses $\Delta z \sim 0.02 R i^{1 / 2} H_{\mathrm{g}} \sim$ $2 \times 10^{9}(\mathrm{Ri} / 0.1)^{1 / 2}(\mathrm{r} / \mathrm{AU})^{9 / 7} \mathrm{~cm}$ considered in this paper. Moreover, the lifetimes of the particle clumps on a given eddy length scale should roughly equal the eddy turnover times, which for the smallest eddies are of or$\operatorname{der} t_{\mathrm{i}} \sim \sqrt{\nu t_{\mathrm{o}}} / \delta v_{\mathrm{o}} \sim 10^{2}(r / \mathrm{AU})^{55 / 28} \mathrm{~s}$. We do not expect such rapid fluctuations in particle density, occurring on such small length scales, to affect significantly the evolution of the slower, larger scale KHI. Turbulent clumping may only serve as a source of noise on tiny scales. The possibility that turbulent clumping could still be significant on larger scales is still being investigated (Hogan \& Cuzzi 2007; Cuzzi et al. 2008).

The perfect coupling approximation prevents us from studying how particles sediment out of gas into dusty sublayers, but it does not stop us from identifying what kinds of sublayers are dynamically stable to the KHI. A subdisk with a given density profile is either dynamically stable or it is not, and we can run the B09 code for many dynamical times (typically 60 or more) to decide the answer. In a forthcoming paper we will combine the B09 code with a settling algorithm that will permit us to study how dust settles from arbitrary initial conditions, freeing us from the assumption that the density profile derives from a constant Richardson number.

\subsection{Organization of this Paper}

Our numerical methods, including our rationale for choosing box sizes and resolutions, are described in $\$ 2$. Results are presented in $\$ 3$ and discussed in $\$ 4$.

\section{METHODS}

The equations solved by the B09 code are rederived in 2.1. Initial conditions for our simulations are given in \$2.2. The code itself is briefly described in $\$ 2.3$ Our choices for box size and resolution are explained in $\$ 2.4$

\subsection{Equations}

The equations we solve are identical to equations (12ae) of B09. We outline their derivation here, filling in steps skipped by B09, adjusting the notation, and providing some clarifications. This section may be skimmed on a first reading.

We begin with the equations for an ideal gas perfectly coupled to pressureless dust in an inertial frame:

$$
\begin{aligned}
\frac{d \mathbf{v}}{d t} & =-\nabla \Phi-\frac{\nabla P}{\rho_{d}+\rho_{g}}, \\
\frac{d \rho_{\mathrm{g}}}{d t} & =-\rho_{g} \nabla \cdot \mathbf{v}, \\
\frac{d\left(\rho_{\mathrm{d}} / \rho_{\mathrm{g}}\right)}{d t} & =0, \\
\rho_{g} C_{\mathrm{V}} \frac{d T}{d t} & =-P \nabla \cdot \mathbf{v}, \\
P & =\Re \rho_{\mathrm{g}} T,
\end{aligned}
$$

where $d / d t$ is the convective derivative, $\rho_{\mathrm{g}(\mathrm{d})}$ is the density of gas (dust), $P$ is the gas pressure, and $T$ is the gas temperature. Under the assumption that they are perfectly coupled, gas and dust share the same velocity $\mathbf{v}$, and the dust-to-gas ratio is conserved in a Lagrangian sense. The background potential is provided by the central star of mass $M: \Phi=-G M / \sqrt{r^{2}+z^{2}}$, where $r$ is the cylindrical radius and $z$ is the vertical distance above the disk midplane. There are five equations for the five flow variables $\mathbf{v}, \rho_{\mathrm{g}}, \rho_{\mathrm{d}}, P$, and $T$. The thermodynamic constants include the specific heat $C_{\mathrm{V}}=\Re /(\gamma-1)$ at constant volume, the ideal gas constant $\Re=C_{\mathrm{P}}-C_{\mathrm{V}}$, the specific heat $C_{\mathrm{P}}$ at constant pressure, and $\gamma=C_{\mathrm{P}} / C_{\mathrm{V}}$. Equation (11) is equivalent to the condition that the flow be isentropic $\left[d\left(P \rho_{\mathrm{g}}^{-\gamma}\right) / d t=0\right]$. The code which solves the fluid equations actually employs an artificial hyperviscosity to damp away the smallest scale perturbations (\$2.3); in writing down equations (8)-(12), we have omitted the hyperviscosity terms for simplicity.

We move to a frame co-rotating with dust-free gas at some fiducial radius $r=R$. This frame has angular frequency $\Omega_{\mathrm{F}}$ given by (1) with $\Omega_{\mathrm{K}}=\left(G M / R^{3}\right)^{1 / 2}$. We define a velocity $v_{\max }$ using the pressure support parameter $\eta$, as given by (2):

$$
\left.v_{\max } \equiv \eta\right|_{r=R} \Omega_{\mathrm{K}} R \text {. }
$$

The velocity $v_{\max }$ is the difference in azimuthal velocity between a strictly Keplerian flow and dust-free gas; it is the maximum possible difference in velocity, attained at large $\mu_{0}$, between gas at the midplane and gas at altitude. The quantities $v_{\max }, \eta$, and the background radial pressure gradient are equivalent; specifying one specifies the other two. Our numerical models are labeled by $v_{\max }$.

In addition to moving into a rotating frame, we also replace the usual cylindrical coordinates $(r, \phi, z)$ with local Cartesian coordinates $x=r-R, y=\left(\phi-\Omega_{\mathrm{F}} t\right) R$, and $z$ Keeping terms to first order in $|x| \sim|z| \sim \eta R$ (see the discussion surrounding equation 6) and dropping

8 Throughout this paper we alternate freely between subscripts $(x, y, z)$ and $(r, \phi, z)$. 
curvature terms, the momentum equation (8) reads

$$
\frac{d \mathbf{v}}{d t}=-2 \Omega_{\mathrm{K}} \hat{\mathbf{z}} \times \mathbf{v}+3 \Omega_{\mathrm{K}}^{2} x \hat{\mathbf{x}}-\Omega_{\mathrm{K}}^{2} z \hat{\mathbf{z}}-\frac{1}{\rho_{\mathrm{d}}+\rho_{\mathrm{g}}} \nabla P-2 \Omega_{\mathrm{K}}^{2} \eta R \hat{\mathbf{x}}
$$

where $d / d t=\partial / \partial t+v_{i} \partial / \partial x_{i}(i=x, y, z)$. On the right-hand side, the first term is the Coriolis acceleration, the second combines centrifugal and radial gravitational accelerations, the third represents the vertical gravitational acceleration from the star, and the last term arises from the centrifugal acceleration in a frame rotating at $\Omega_{\mathrm{F}} \neq \Omega_{\mathrm{K}}$. The remaining fluid equations appear the same as (9)-(12), except that $\mathbf{v}$ is now measured in a (rigidly) rotating frame.

We measure all flow variables relative to a timeindependent reference state (subscripted "ref"):

$$
\begin{gathered}
\mathbf{v}=\mathbf{v}_{\text {ref }}+\widetilde{\mathbf{v}}=\widetilde{\mathbf{v}} \\
P=P_{\text {ref }}+\widetilde{P} \\
\rho_{\mathrm{g}}=\rho_{\mathrm{g}, \text { ref }}+\widetilde{\rho}_{\mathrm{g}} \\
T=T_{\text {ref }}+\widetilde{T} \\
\rho_{\mathrm{d}}=\rho_{\mathrm{d}, \mathrm{ref}}+\widetilde{\rho}_{\mathrm{d}}=\widetilde{\rho}_{\mathrm{d}} .
\end{gathered}
$$

The reference state is defined as follows. It is dust-free $\left(\rho_{\mathrm{d}, \mathrm{ref}}=0\right)$ and has constant gas temperature $T_{\text {ref }}$. The gas in the reference state does not shear, either in the radial or vertical directions, but rotates with a fixed angular frequency $\Omega_{\mathrm{F}}$ in the inertial frame (hence $\mathbf{v}_{\text {ref }}=0$ in the rotating frame). In the reference state there exists a radial pressure gradient directed outward

$$
-\frac{1}{\rho_{\mathrm{g}, \mathrm{ref}}} \frac{\partial P_{\mathrm{ref}}}{\partial r}=2 \Omega_{\mathrm{K}}^{2} \eta R=2 \Omega_{\mathrm{K}} v_{\max }
$$

and a vertical pressure gradient balanced by vertical tidal gravity

$$
-\frac{1}{\rho_{\mathrm{g}, \mathrm{ref}}} \frac{\partial P_{\mathrm{ref}}}{\partial z}=\Omega_{\mathrm{K}}^{2} z
$$

Equation (16) together with equation (12) and the assumption of constant $T_{\text {ref }}$ implies that the reference gas density $\rho_{\mathrm{g}, \text { ref }}$ and pressure $P_{\text {ref }}$ have Gaussian vertical distributions in $z$ with scale height $H_{\mathrm{g}}=\sqrt{\Re T_{\text {ref }}} / \Omega_{\mathrm{K}}$. For simplicity we neglect the radial density gradient $\left(\partial \rho_{\mathrm{g}, \mathrm{ref}} / \partial r=0\right)$, as did B09. This reference state should not be confused with our equilibrium states of interest (\$2.2), which do shear and which do contain dust. The reference state merely serves as a fiducial.

The flows of interest are subsonic. Mach numbers $\epsilon \equiv$ $\widetilde{v} / c_{\mathrm{s}}$ peak at $v_{\max } / c_{\mathrm{s}} \sim c_{\mathrm{s}} /\left(\Omega_{\mathrm{K}} R\right) \sim 0.02$ for gas sound speeds $c_{\mathrm{s}} \sim 1 \mathrm{~km} / \mathrm{s}$ at $R \sim 1 \mathrm{AU}$. Such flow is nearly incompressible: $\left|\widetilde{\rho}_{\mathrm{g}}\right| / \rho_{\mathrm{g}, \text { ref }} \sim|\widetilde{P}| / P_{\text {ref }} \sim|\widetilde{T}| / T_{\text {ref }} \sim \epsilon^{2}$. Invoking the anelastic approximation, we keep only terms leading in $\epsilon$ in any given equation. Equations (9), (10), and (12) reduce to:

$$
\begin{gathered}
\frac{d \rho_{\mathrm{g}}}{d t}+\rho_{\mathrm{g}} \nabla \cdot \mathbf{v}=\frac{\partial \rho_{\mathrm{g}}}{\partial t}+\nabla \cdot\left(\rho_{\mathrm{g}} \mathbf{v}\right) \approx \nabla \cdot\left(\rho_{\mathrm{g}, \mathrm{ref}} \mathbf{v}\right)=0 \\
\frac{d\left(\rho_{\mathrm{d}} / \rho_{\mathrm{g}}\right)}{d t} \approx \frac{d\left(\widetilde{\rho_{\mathrm{d}}} / \rho_{\mathrm{g}, \text { ref }}\right)}{d t} \equiv \frac{d \mu}{d t}=0 \\
\frac{\widetilde{P}}{\rho_{\mathrm{g}, \text { ref }}} \equiv \widetilde{h}=\frac{\widetilde{\rho_{\mathrm{g}}}}{\rho_{\mathrm{g}, \text { ref }}} \Re T_{\text {ref }}+\Re \widetilde{T}
\end{gathered}
$$

where we define $\mu \equiv \widetilde{\rho}_{\mathrm{d}} / \rho_{\mathrm{g}, \text { ref }}=\rho_{\mathrm{d}} / \rho_{\mathrm{g} \text {,ref }}$ and the pressure-like enthalpy $\widetilde{h} \equiv \widetilde{P} / \rho_{\text {g,ref }}$, and henceforth for convenience drop all tildes on $\rho_{\mathrm{d}}, \mu$, and $\mathbf{v}$ (but not the other variables related to gas). The rightmost equalities of (17), (18), and (19) match equations (12b), (12c), and (12e) of B09. The anelastic approximation has been employed in the study of atmospheric convection (Ogura \& Phillips 1962; Gough 1969), stars (Gilman \& Glatzmaier 1981), and vortices in protoplanetary disks (Barranco \& Marcus 2000, 2005, 2006). By eliminating the time derivative in the continuity equation (17), we effectively "sound-proof" the fluid. The simulation timestep is not limited by the sound-crossing time but rather by the longer advection time.

We rewrite our energy equation (11) as follows: replace $-\nabla \cdot \mathbf{v}$ with $d \ln \rho_{\mathrm{g}} / d t=-d \ln T / d t+d \ln P / d t$ to find that

$$
\begin{aligned}
C_{\mathrm{P}} \frac{d \widetilde{T}}{d t} & =\frac{1}{\rho_{\mathrm{g}}} \frac{d P}{d t} \\
& \approx \frac{1}{\rho_{\mathrm{g}, \mathrm{ref}}} \mathbf{v} \cdot \nabla P_{\mathrm{ref}} \\
& \approx-\mathbf{v} \cdot\left(2 \Omega_{\mathrm{K}}^{2} \eta R \hat{\mathbf{x}}+\Omega_{\mathrm{K}}^{2} z \hat{\mathbf{z}}\right)
\end{aligned}
$$

where for the second line we dropped $d \widetilde{P} / d t$ in comparison to $\mathbf{v} \cdot \nabla P_{\text {ref }}$, and for the third line we replaced $\rho_{\mathrm{g}, \mathrm{ref}}^{-1} \nabla P_{\text {ref }}$ using (15) and (16). Equation (20) matches (12d) of B09 except that for the right-hand side he has a coefficient equal to $1+\widetilde{T} / T_{\text {ref }}$, which we have set to unity.

Finally, to recover the form of the momentum equation (12a) of B09, first consider the pressure acceleration and isolate the contribution from dust-free gas $\left(-\rho_{\mathrm{g}}^{-1} \nabla P\right)$ :

$$
\begin{aligned}
-\frac{1}{\rho_{\mathrm{d}}+\rho_{\mathrm{g}}} \nabla P & =-\left(\frac{1}{\rho_{\mathrm{d}}+\rho_{\mathrm{g}}}-\frac{1}{\rho_{\mathrm{g}}}\right) \nabla P-\frac{1}{\rho_{\mathrm{g}}} \nabla P \\
& \approx \frac{\mu}{\mu+1}\left(\frac{1}{\rho_{\mathrm{g}}} \nabla P\right)-\frac{1}{\rho_{\mathrm{g}}} \nabla P .
\end{aligned}
$$

Now expand

$$
\begin{aligned}
\frac{1}{\rho_{g}} \nabla P & \approx \frac{1}{\rho_{\mathrm{g}, \text { ref }}} \nabla P_{\text {ref }}+\frac{1}{\rho_{\mathrm{g}, \text { ref }}} \nabla \widetilde{P}-\frac{\widetilde{\rho_{\mathrm{g}}}}{\rho_{\mathrm{g}, \text { ref }}} \nabla P_{\text {ref }} \\
& \approx \frac{1}{\rho_{\mathrm{g}, \text { ref }}} \nabla P_{\text {ref }}+\nabla \widetilde{h}+\frac{\widetilde{T}}{T_{\text {ref }}}\left(\frac{\nabla P_{\text {ref }}}{\rho_{\mathrm{g}, \text { ref }}}\right) \\
& \approx-\left(1+\frac{\widetilde{T}}{T_{\text {ref }}}\right)\left(2 \Omega_{\mathrm{K}}^{2} \eta R \hat{\mathbf{x}}+\Omega_{\mathrm{K}}^{2} z \hat{\mathbf{z}}\right)+\nabla \widetilde{h}
\end{aligned}
$$


where for the last line we used (15) and (16). Insertion of (21) and (22) into (14) yields the anelastic momentum equation (12a) of B09:

$$
\begin{aligned}
\frac{d \mathbf{v}}{d t}= & -2 \Omega_{\mathrm{K}} \hat{\mathbf{z}} \times \mathbf{v}+3 \Omega_{\mathrm{K}}^{2} x \hat{\mathbf{x}}+\frac{\widetilde{T}}{T_{\mathrm{ref}}}\left(2 \Omega_{\mathrm{K}}^{2} \eta R \hat{\mathbf{x}}+\Omega_{\mathrm{K}}^{2} z \hat{\mathbf{z}}\right)-\nabla \widetilde{h} \\
& -\frac{\mu}{\mu+1}\left[\left(1+\frac{\widetilde{T}}{T_{\text {ref }}}\right)\left(2 \Omega_{\mathrm{K}}^{2} \eta R \hat{\mathbf{x}}+\Omega_{\mathrm{K}}^{2} z \hat{\mathbf{z}}\right)-\nabla \widetilde{h}\right] .
\end{aligned}
$$

which isolates the driving term due to dust.

\subsection{Initial Conditions}

Equilibrium initial conditions (superscripted " $\nmid$ ") are specified by five functions: $\mu=\mu^{\dagger}, \widetilde{T}=\widetilde{T}^{\dagger}, \widetilde{h}=\widetilde{h}^{\dagger}$, $\widetilde{\rho_{\mathrm{g}}}={\widetilde{\rho_{\mathrm{g}}}}^{\dagger}$, and $\mathbf{v}=\mathbf{v}^{\dagger}$. For $\mu^{\dagger}$, we use flows characterized by a globally constant Richardson number (Sekiva 1998; Youdin \& Shu 2002; Chiang 2008). The conditions $R i=$ constant, $\partial \rho_{\mathrm{g}} / \partial z \ll \partial \rho_{\mathrm{d}} / \partial z$, and $g=-\Omega_{\mathrm{K}}^{2} z$ (no selfgravity) yield

$$
\mu^{\dagger}(z)=\left[\frac{1}{1 /\left(1+\mu_{0}\right)^{2}+\left(z / z_{\mathrm{d}}\right)^{2}}\right]^{1 / 2}-1,
$$

where $\mu_{0}$ is the initial midplane dust-to-gas ratio and

$$
z_{\mathrm{d}} \equiv \frac{R i^{1 / 2} v_{\max }}{\Omega_{\mathrm{K}}}
$$

is a characteristic dust height. The dust density peaks at the midplane and decreases to zero at

$$
z= \pm z_{\max }= \pm \frac{\sqrt{\mu_{0}\left(2+\mu_{0}\right)}}{1+\mu_{0}} z_{\mathrm{d}}
$$

which is consistent with our order-of-magnitude expression (6). Neither equation (24) nor the code accounts for self-gravity and therefore we are restricted to modeling flows whose densities are less than that required for the Toomre parameter of the subdisk to equal unity (CY10; see also (4). For the minimum-mass disk of CY10, this restriction is equivalent to $\mu \lesssim 30$. Input model parameters include $\mu_{0}, R i$, and $v_{\max }$.

For the gas, we assume

$$
\widetilde{T}^{\dagger}=0
$$

(initially isothermal) and solve vertical hydrostatic equilibrium for $\widetilde{h}^{\dagger}$ (the $z$-component of equation 23):

$$
\frac{\partial \widetilde{h}^{\dagger}}{\partial z}=-\mu^{\dagger} \Omega_{\mathrm{K}}^{2} z
$$

The functional form for $\widetilde{h}^{\dagger}(z)$ is not especially revealing and so we do not write it out here. For simplicity we assume that $\widetilde{h}^{\dagger}$ does not depend on $x$. From $\widetilde{h}^{\dagger}$ and $\widetilde{T}^{\dagger}=0$ it follows from (19) that

$$
{\tilde{\rho_{\mathrm{g}}}}^{\dagger}=\frac{\rho_{\mathrm{g}, \mathrm{ref}} \widetilde{h}^{\dagger}}{\Re T_{\mathrm{ref}}} .
$$

The fractional deviations ${\widetilde{\rho_{\mathrm{g}}}}^{\dagger} / \rho_{\mathrm{g} \text {,ref }}$ and $\widetilde{P}^{\dagger} / P_{\text {ref }}$ from the reference state are very small, of order $\mu^{\dagger}\left(v_{\max } / c_{\mathrm{s}}\right)^{2} R i$.
It remains to specify $\mathbf{v}^{\dagger}$. Using the conditions on $\widetilde{h}^{\dagger}$ stated above, we solve for the equilibrium (steady-state) solution to equation (23):

$$
\begin{aligned}
& v_{x}^{\dagger}=v_{z}^{\dagger}=0 \\
& v_{y}^{\dagger}=-\frac{3}{2} \Omega_{\mathrm{K}} x+\left[\frac{\mu^{\dagger}(z)}{\mu^{\dagger}(z)+1}\right] v_{\max } .
\end{aligned}
$$

In our reference frame rotating with the velocity of dustfree gas at $R$, the first term on the right side of (30) accounts for the standard Kepler shear, while the second term describes how dust, which adds to inertia but not pressure, speeds up the gas.

To $\mu^{\dagger}$ we add random perturbations

$$
\Delta \mu(x, y, z)=A(x, y) \mu^{\dagger}(z)\left[\cos \left(\pi z / 2 z_{d}\right)+\sin \left(\pi z / 2 z_{d}\right)\right] .
$$

The amplitude $A(x, y)$ is constructed in Fourier space so that each Fourier mode has a random phase and an amplitude inversely proportional to the horizontal wavenumber: $\hat{A} \propto k_{\perp}^{-1}=\left(k_{x}^{2}+k_{y}^{2}\right)^{-1 / 2}$. Because our box sizes are scaled to $z_{\max }$, our Fourier noise amplitudes are largest on scales comparable to the dust layer thickness. Thus those modes which are most likely to overturn the layer are given the greatest initial power. The perturbations are also chosen to be antisymmetric about the $x$-axis so that no extra energy is injected into the system. We take the root-mean-squared amplitude $A_{\mathrm{rms}} \equiv\left\langle A^{2}\right\rangle^{1 / 2}$ of the perturbations to be $10^{-4}$ or $10^{-3}$.

In summary, three input parameters $\mu_{0}, R i$, and $v_{\max }$ determine our isothermal equilibrium initial conditions (equations 24, 28, and 30) 9] The equilibrium solution for $\mu(z)$ is then perturbed (equation 31) by a root-meansquared fractional amount $A_{\text {rms }}$. The parameters of primary interest are $\mu_{0}$ and $R i$. For the remaining parameters $v_{\max }$ and $A_{\text {rms }}$ we consider three possible combinations: $\left(v_{\max }, A_{\mathrm{rms}}\right)=\left(0.025 c_{\mathrm{s}}, 10^{-4}\right)$ for our standard runs; $\left(0.025 c_{\mathrm{s}}, 10^{-3}\right)$ to probe larger initial perturbations; and $\left(0.05 c_{\mathrm{s}}, 10^{-4}\right)$ to assess the effect of a stronger radial pressure gradient.

Note that specifying $\mu_{0}$ and $R i$ (and $v_{\max }$, though this last variable is fixed for all of our standard runs) specifies the entire dust and gas vertical profiles, $\rho_{\mathrm{d}}(z)$ and $\rho_{\mathrm{g}}(z)$, and by extension the bulk height-integrated metallicity, $\Sigma_{\mathrm{d}} / \Sigma_{\mathrm{g}} \equiv \int \rho_{\mathrm{d}} d z / \int \rho_{\mathrm{g}} d z$. We do not give an explicit expression for $\Sigma_{\mathrm{d}} / \Sigma_{\mathrm{g}}$ because it is cumbersome and not particularly revealing. The bulk metallicity is in some sense the most natural independent variable because its value is given by the background disk (for ways in which the bulk metallicity may change, e.g., by radial particle drifts, see CY10). We will plot our results in the space of $\mu_{0}, R i$, and $\Sigma_{\mathrm{d}} / \Sigma_{\mathrm{g}}$, keeping in mind that only two of these three variables are independent.

\subsection{Code}

We use the spectral, anelastic, shearing box code developed by Barranco \& Marcus (2006) and modified by

\footnotetext{
9 While our initial conditions are isothermal, the temperature of the flow can change because of adiabatic compression/expansion and because our artificial hyperviscosity dissipates the highest wavenumber disturbances. These temperature changes are fractionally tiny because the flow is highly subsonic.
} 
B09 to simulate well-coupled gas and dust. The code employs shearing periodic boundary conditions in $r$, periodic boundary conditions in $\phi$, and closed lid boundaries in $z$; the vertical velocity $v_{z}$ is required to vanish at the top and bottom of the box $\left(z= \pm L_{z} / 2\right)$.

Spectral methods approximate the solution to the fluid equations as a linear combination of basis functions. The basis functions describe how the flow varies in space, and the coefficients of the functions are determined at every timestep. For each of the periodic dimensions, a standard Fourier basis is used, while for the vertical direction, Chebyshev polynomials are employed. Whereas in $r$ and $\phi$ grid points are evenly spaced, the use of Chebyshev polynomials in $z$ has the effect that vertical grid points are unevenly spaced; points are concentrated towards the top and bottom boundaries of the box, away from the midplane where the dust layer resides. Thus to resolve the dust layer vertically, we need to increase the number of vertical grid points $N_{z}$ by an amount disproportionately large compared to the numbers of radial and azimuthal grid points $N_{r}$ and $N_{\phi}$. See $₫ 2.4$ for further discussion.

Spectral codes have no inherent grid dissipation; energy is allowed to cascade down to the smallest resolved length scales through nonlinear interactions. To avoid an energy "pile-up" at the highest wavenumbers, we dissipate energy using an artificial hyperviscosity, given in $\S 3.3 .3$ of Barranco \& Marcus (2006).

Simulations satisfy the Courant-Friedrichs-Lewy (CFL) condition which states that the CFL number, defined as the code timestep divided by the shortest advection time across a grid cell, be small. In the shearing coordinates in which the code works, that advection time is the cell dimension divided by the local velocity over and above the Keplerian shear, i.e., orbital velocities are subtracted off before evaluating the CFL number. All simulations reported in this paper are characterized by CFL numbers less than about 0.1 .

\subsection{Box Size and Numerical Resolution}

Our standard box dimensions are $\left(L_{r}, L_{\phi}, L_{z}\right)=$ $(6.4,12.8,8) z_{\max }$ and the corresponding numbers of grid points are $\left(N_{r}, N_{\phi}, N_{z}\right)=(32,64,128)$. By scaling our box lengths $L_{i}$ to $z_{\max }$ and fixing the numbers of grid points $N_{i}$, we ensure that each standard simulation enjoys the same resolution (measured in grid points per physical length) regardless of $R i, \mu_{0}$, and $v_{\max }$. The vertical extent of the dust layer between $z= \pm z_{\max }$ is resolved by 22 grid points (this is less than $\left[128 /\left(8 z_{\max }\right)\right] \times$ $2 z_{\max }=32$ because the Chebyshev-based vertical grid only sparsely samples the midplane). The radial and azimuthal directions are resolved by 10 grid points per $2 z_{\max }$ length. We choose our resolution in the vertical direction to be greater than that of the horizontal directions because the dust layer has finer scale structure in $z$ : the dust layer becomes increasingly cuspy at the midplane as $\mu_{0}$ increases. We prescribe the same resolution in the radial and azimuthal directions $\left(L_{\phi} / N_{\phi}=L_{r} / N_{r}\right)$; experiments with different resolutions in $r$ and $\phi$ generated spurious results.

Too small a box size can artificially affect the stability of the dust layer, because a given box can only support modes having integer numbers of wavelengths inside it. Small boxes may be missing modes that in reality over-

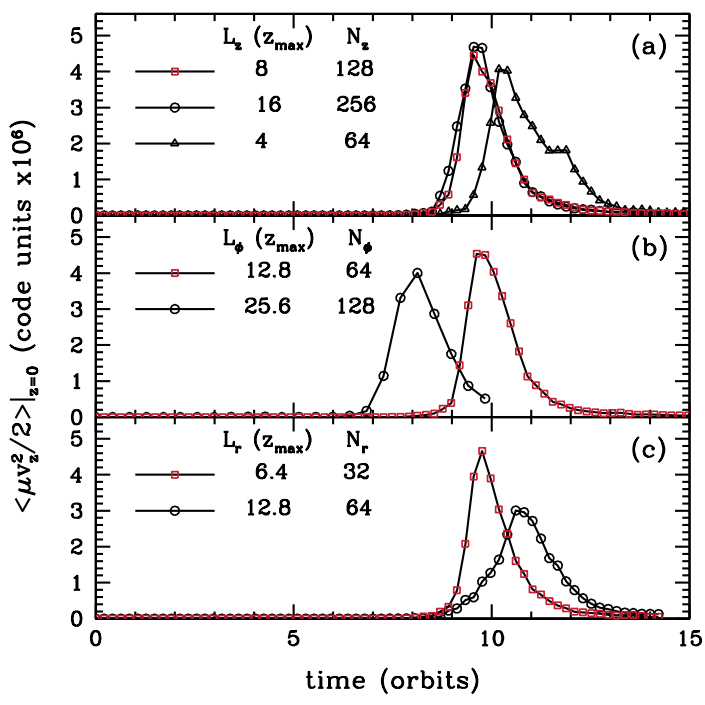

FIG. 1. - Testing box sizes at fixed numerical resolution. For our standard box, $\left(L_{r}, L_{\phi}, L_{z}\right)=(6.4,12.8,8) z_{\max }$ and $\left(N_{r}, N_{\phi}, N_{z}\right)=(32,64,128)$. In each panel we vary one box dimension while keeping the other two dimensions fixed at their standard values. In the top panel we vary $L_{z}$ at fixed resolution $N_{z} / L_{z}$. In the middle and bottom panels, $L_{\phi}$ and $L_{r}$ are varied in turn. All simulations in this figure have $\mu_{0}=10, R i=0.1, v_{\max }=0.025 c_{\mathrm{S}}$, $A_{\mathrm{rms}}=10^{-4}$, and use code units $\rho_{\mathrm{g}, \mathrm{ref}}(z=0)=\Omega_{\mathrm{K}}=H_{\mathrm{g}}=1$. Doubling the box dimensions from our standard values changes when the average vertical kinetic energy peaks by only a few orbits at most. The average \langle\rangle is performed over all $r$ and $\phi$ at fixed $z=0$.

turn the layer. We verify that for all runs in which the dust layer overturns, the KH mode that most visibly disrupts the layer spans more than one azimuthal wavelength. Typically $3-5$ wavelengths are discerned across the box.

To more thoroughly test our standard choices for $L_{i}$, we study how systematic variations in box length affect how the instability develops. For this test, we adopt a fixed set of physical input parameters, $\left(R i, \mu_{0}, v_{\max }\right)=\left(0.1,10,0.025 c_{s}\right)$, which should lead to instability (Chiang 2008). Our diagnostic is the time evolution of the vertical kinetic energy at the midplane: $\left\langle\mu(t) v_{z}^{2}(t)\right\rangle / 2$, where the average is over all $r$ and $\phi$ at fixed $z=0$ and time $t$. We vary $L_{i}$ and $N_{i}$ in tandem to maintain the same resolution from run to run, thereby isolating the effect of box size. Figure 1 shows how doubling one of the box dimensions while fixing the other two alters the time history of $\left\langle\mu v_{z}^{2}\right\rangle / 2$. Panel (a) demonstrates that our standard choice for $L_{z}=8 z_{\max }$ is sufficiently large because the curves for $L_{z}=8 z_{\max }$ and $L_{z}=16 z_{\text {max }}$ practically overlap. Panels (b) and (c) show that our standard choices for $L_{\phi}=12.8 z_{\max }$ and $L_{r}=16 z_{\max }$ are somewhat less adequate. The peak of the curve for $\left(L_{\phi}, N_{\phi}\right)=\left(12.8 z_{\max }, 64\right)$ is delayed by two orbits compared to that for $\left(L_{\phi}, N_{\phi}\right)=\left(25.6 z_{\max }, 128\right)$, and the curve for $\left(L_{r}, N_{r}\right)=\left(6.4 z_{\max }, 32\right)$ peaks an orbit earlier than that for $\left(L_{r}, N_{r}\right)=\left(12.8 z_{\max }, 64\right)$. Nevertheless these time differences are small compared to the total time to instability, about 10 orbits. Moreover, the errors point in opposite directions. Thus we expect our choices for $L_{\phi}$ and $L_{r}$ to partially compensate for each other so that any error due to our box size in calculating the time to instability will be less than $\sim 1$ orbit. 
We test how robust our results are to numerical resolution by re-running a few simulations at twice the normal resolution (doubling $N_{i}$ while fixing $L_{i}$ ). Results at high resolution are given in $\$ 3.3$. Every simulation is run for at least ten orbits. A typical run performed at our standard resolution takes approximately 2.5 wall-clock hours using 56 processors on the Purdue Steele cluster. A highresolution run takes about 32 wall-clock hours.

\section{RESULTS}

In our standard simulations, we fix $v_{\max }$ and $A_{\text {rms }}$ while systematically varying $R i$ and $\mu_{0}$ from run to run. Our systematic variations of $R i$ and $\mu_{0}$ correspond to systematic variations in $\Sigma_{\mathrm{d}} / \Sigma_{\mathrm{g}}$; recall that only two of the three parameters $R i, \mu_{0}$, and $\Sigma_{\mathrm{d}} / \Sigma_{\mathrm{g}}$ are independent. For each $\mu_{0} \in\{0.3,1,3,10\}$ we adjust $R i$ until the threshold value $R i_{\text {crit }}$ dividing stable from unstable runs is determined to within 0.1 dex.

Deciding by numerical simulation whether a given dust layer is stable or not is unavoidably subject to the finite duration of the simulation. We define our criteria for deciding stability in $\$ 3.1$, Results are given in $\$ 3.2$ and tested for robustness in 3.3 .

\subsection{Criteria for Stability}

Stability is assessed by two quantities: the midplane vertical kinetic energy

$$
\left\langle\mu v_{z}^{2}\right\rangle / 2 \text { as a function of } t
$$

where the average is performed over $r$ and $\phi$ at fixed $z=0$ and $t$, and the dust density profile

$$
\langle\mu\rangle \text { as a function of } z \text { and } t
$$

where the average is performed over $r$ and $\phi$ at fixed $z$ and $t$. By definition, in an "unstable" run, $\left\langle\mu v_{z}^{2}\right\rangle / 2$ grows exponentially over several orbital periods, and $\langle\mu\rangle$ deviates from its initial value $\left\langle\mu^{\dagger}\right\rangle$ by more than $15 \%$. "Stable" simulations satisfy neither criterion. Some runs are "marginally unstable" in that they satisfy the first but not the second criterion. At the end of the standard ten-orbit duration of a marginally unstable run, we find the kinetic energy continues to rise, suggesting that were the run to be extended for longer than ten orbits, the dust layer would eventually overturn. In every instance where we extend the duration of a marginally unstable run, we verify that this is the case. Thus "marginally unstable" is practically synonymous with "unstable."

Examples of unstable and stable runs are shown in Figure 2. In the unstable simulation, after $t \approx 6$ orbits, the kinetic energy rises exponentially. At $t \approx 9$ orbits, the dust layer overturns and the midplane dust-to-gas ratio falls by more than $60 \%$. By contrast, in the stable simulation, after an initial adjustment period lasting $\sim 3$ orbits during which the midplane value of $\langle\mu\rangle$ decreases by $10 \%$, the kinetic energy drops by orders of magnitude to a nearly constant value and shows no evidence of further growth.

Figure 3 shows the evolution of $\left|v_{i}(z)\right|(i=r, \phi, z)$ and $\langle\mu(z)\rangle$ for the same unstable run of Figure 2, The velocity data are sampled at a single $(x, y)$ position at the center of our simulation box. The radial and vertical velocities $\left|v_{r}\right|$ and $\left|v_{z}\right|$, initially zero, grow to become comparable with the shearing velocity $\left|v_{\phi}\right|$. Figure 4 displays corresponding snapshots of $\mu(y, z)$, taken at a single radius $x$ near the center of our box. Though the data in Figures 3 and 4 are sampled at particular radial locations in our box, we verify that the instability develops similarly at all locations - as it should - unlike the ZEUS-based simulations of Chiang (2008).

\subsection{Stability as a Function of Ri, $\mu_{0}$, and $\Sigma_{\mathrm{d}} / \Sigma_{\mathrm{g}}$}

Figure 5 maps the stable and unstable regions in $\left(R i, \mu_{0}\right)$ space, for fixed $v_{\max }=0.025 c_{\mathrm{s}}$ and $A_{\mathrm{rms}}=10^{-4}$. Figures 6 and 7 portray the same data using alternate but equivalent projections of parameter space: $\left(R i, \Sigma_{\mathrm{d}} / \Sigma_{\mathrm{g}}\right)$ and $\left(\mu_{0}, \Sigma_{\mathrm{d}} / \Sigma_{\mathrm{g}}\right)$, respectively.

These plots demonstrate that there is no unique value of $R i_{\text {crit }}$. Rather $R i_{\text {crit }}$ is a function of $\mu_{0}$, or equivalently a function of $\Sigma_{\mathrm{d}} / \Sigma_{\mathrm{g}}$. For bulk metallicities $\Sigma_{\mathrm{d}} / \Sigma_{\mathrm{g}}$ near the solar value, $R i_{\text {crit }}$ is found to be close to the classical value of $1 / 4$. But as $\Sigma_{\mathrm{d}} / \Sigma_{\mathrm{g}}$ decreases below the solar value, $R i_{\text {crit }}$ shrinks to $\sim 0.01$ or even lower. A leastsquares fit to the four midpoints (evaluated in log space) in Figure 5] dividing neighboring stable points (in black) and unstable points (in red or red outlined with black) yields $R i_{\text {crit }} \propto \mu_{0}^{1.0}$. This same fit, projected into metallicity space, is shown in Figures [6 and 7] in metallicity space the stability boundary is not a power law.

As Figure 7 attests, dust-to-gas ratios $\mu_{0}$ as high as $\sim 8$ can be attained in disks of solar metallicity without triggering a shear instability: see the intersection between the dashed curve fitted to our standard resolution data, and the dotted line representing solar metallicity. This intersection occurs at $\mu_{0} \approx 7$. Were we to re-fit the dashed curve using the higher resolution data represented by triangles, the intersection with solar metallicity would occur at $\mu_{0}$ closer to 8 .

A dust-to-gas ratio of $\mu_{0} \approx 8$ is within a factor of $\sim 4$ of the Toomre threshold for gravitational fragmentation in a minimum-mass disk (CY10; 4 ). We can achieve the Toomre threshold by simply allowing for a gas disk that is $\sim 4 \times$ more massive than the minimummass nebula. Alternatively we can enrich the disk in metals to increase $\Sigma_{\mathrm{d}} / \Sigma_{\mathrm{g}}$. Extrapolating the boundary of stability (dashed curve) in Figure 7 to higher $\Sigma_{\mathrm{d}} / \Sigma_{\mathrm{g}}$ suggests that the Toomre threshold $\mu_{0} \approx 30$ could be achieved for minimum-mass disks having $\sim 3 \times$ the solar metallicity. The sensitivity to metallicity is also exemplified by Figure 2. For the same $\mu_{0}=10$, the dust layer based on a near-solar metallicity of $\Sigma_{\mathrm{d}} / \Sigma_{\mathrm{g}}=0.013$ overturns, whereas one derived from a supersolar metallicity of $\Sigma_{\mathrm{d}} / \Sigma_{\mathrm{g}}=0.030$ remains stable.

\subsection{Tests at Higher Resolution, Higher $A_{\text {rms }}$, and Higher $v_{\max }$}

We test how robust our determination of $R i_{\text {crit }}$ is to numerical resolution by redoing our simulations for $\mu_{0}=$ 0.3 and 10 with double the number of grid points in each dimension. The results are overlaid as blue triangles in Figures 5, 6, and [7, At $\mu_{0}=0.3$, increasing the resolution does not change $R i_{\text {crit }}$ from its value of 0.009 . At $\mu_{0}=$ $10, R i_{\text {crit }}$ shifts downward from 0.3 to 0.2 . Although we have not strictly demonstrated convergence of our results with resolution, and although high resolution data at other values of $\mu_{0}$ are missing, it seems safe to conclude 


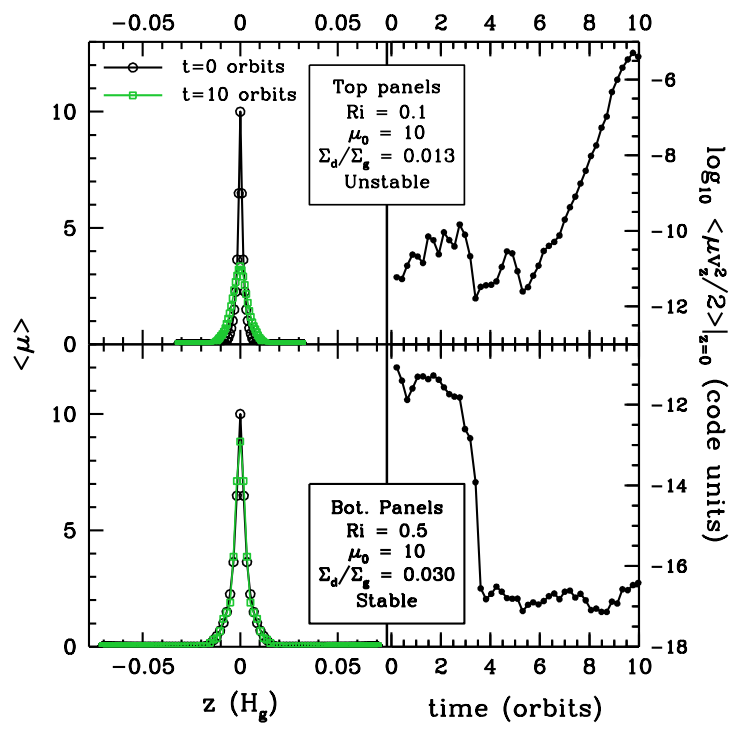

Fig. 2.- Sample unstable (top) and stable (bottom) dust layers. In the unstable case, the layer overturns and mixes dust-rich gas with dust-poor gas, causing the dust-to-gas ratio at the midplane to drop by a factor of $\sim 3$ after 10 orbits (top left). As the instability unfolds, the vertical kinetic energy amplifies exponentially from $t \approx 5-10$ orbits (top right). At fixed $\mu_{0}$, the layer is stabilized by increasing the Richardson number or equivalently the height-integrated metallicity $\Sigma_{\mathrm{d}} / \Sigma_{\mathrm{g}}$. In the stable run, the dust profile changes by less than $15 \%$ (bottom left) while the kinetic energy, after dropping precipitously, shows no indication of growing (bottom right). The two runs shown use $v_{\max }=0.025 c_{\mathrm{s}}$ and $A_{\mathrm{rms}}=10^{-4}$.

that the slope of the stability boundary in $R i-\mu_{0}$ space is close to, but decidedly shallower than, linear.

We also test the sensitivity of our results to $A_{\text {rms }}$. Increasing $A_{\text {rms }}$ by an order of magnitude to $10^{-3}$ shifts $R i_{\text {crit }}$ upward by $\lesssim 0.2$ dex at $\mu_{0}<1$, but leaves $R i_{\text {crit }}$ unchanged at larger $\mu_{0}$ (Figure 8). B09 also reported some sensitivity to $A_{\text {rms }}$.

Tests where $v_{\max }$ was doubled to $0.05 c_{\mathrm{s}}$ reveal no change in $R i_{\text {crit }}$ (data not shown).

\section{SUMMARY AND DISCUSSION}

Where a protoplanetary disk is devoid of turbulence intrinsic to gas, dust particles settle toward the midplane, accumulating in a sublayer so thin and so dense that the dust-gas mixture becomes unstable. If the first instability to manifest is self-gravitational, dust particles are drawn further together, possibly spawning planetesimals. If instead the layer is first rendered unstable by a Kelvin-Helmholtz-type shearing instability (KHI), the resultant turbulence prevents dust from settling further, pre-empting gravitational collapse. In this paper we investigated the conditions which trigger the KHI, hoping to find a region of parameter space where the KHI might be held at bay so that planetesimals can form by selfgravity.

A fundamental assumption underlying our work is that turbulence intrinsic to gas can, in some regions of the disk, be neglected. There is some consensus that near disk midplanes, in a zone extending from $\sim 1$ to at least $\sim 10$ AUs from the parent star, gas may be too poorly ionized to sustain magnetohydrodynamic turbulence (Ilgner \& Nelson 2006; Bai \& Goodman 2009; Turner et al. 2010). Presumably if the magnetorota-

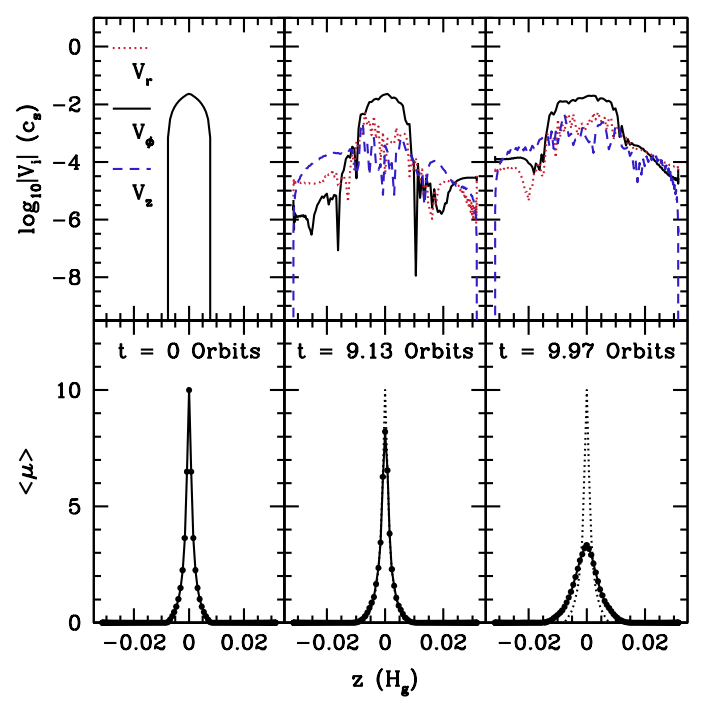

FIG. 3.- Snapshots of absolute values of the three velocity components (top panels) and horizontally averaged dust-to-gas ratio (bottom panels), both as functions of height, at three instants in time. For this unstable run, $\left(R i, \mu_{0}, v_{\max }, A_{\mathrm{rms}}\right)=$ $\left(0.1,10,0.025 c_{\mathrm{s}}, 10^{-4}\right)$. Velocities are taken from a grid point near the middle of the box. The vertical shear $\partial v_{\phi} / \partial z$ inside the dust layer weakens with time as dust is more uniformly mixed with gas, and as the radial and vertical velocities grow at the expense of the azimuthal velocity.

tional instability (e.g., Balbus 2009) cannot operate at the midplane, disk gas there is laminar-pending the uncertain ability of magnetically active surface layers to stir the disk interior (e.g., Turner et al. 2010), or the discovery of a purely hydrodynamic form of turbulence (Lithwick 2009). To get a sense of how laminar disk gas must be to permit dust sublayers to form, Chiang \& Youdin (2010) compared the height to which dust particles are stirred in an "alpha"-turbulent disk to the thickness of the sublayer (6). They estimated that the former is smaller than the latter when the dimensionless turbulent diffusivity $\alpha \lesssim 3 \times 10^{-4} \Omega_{\mathrm{K}} t_{\mathrm{stop}}(r / \mathrm{AU})^{4 / 7}$ for $t_{\text {stop }}<\Omega_{\mathrm{K}}^{-1}$. To place this requirement in context, $\alpha$ values for magnetically active zones are typically quoted to be greater than $\sim 10^{-3}$. Whether magnetically dead zones are sufficiently passive for dust to settle into sublayers remains an outstanding question.

Modulo this concern, we studied the stability of dust layers characterized by spatially constant Richardson numbers $R i$ using a three-dimensional, spectral, anelastic, shearing box code (Barranco \& Marcus 2006) that models gas and dust as two perfectly coupled fluids (Barranco 2009). We found that stability is not characterized by a single critical Richardson number. Rather the value of $R i_{\text {crit }}$ distinguishing layers that overturn from those that do not is a nearly linear function of the midplane dust-to-gas ratio $\mu_{0}$ (Figure 5). Dust-rich sublayers having $\mu_{0} \approx 10$ have $R i_{\text {crit }} \approx 0.2$ - near the canonical value of $1 / 4$ - while dust-poor sublayers having $\mu_{0} \approx 0.3$ (still orders of magnitude dustier than wellmixed gas and dust at solar abundances) have $R i_{\text {crit }}$ as low as 0.009 .

Previous studies (e.g., Sekiva 1998; Youdin \& Shu 2002; Youdin \& Chiang 2004) assumed a universal critical Richardson number of $1 / 4$. This popular assumption 


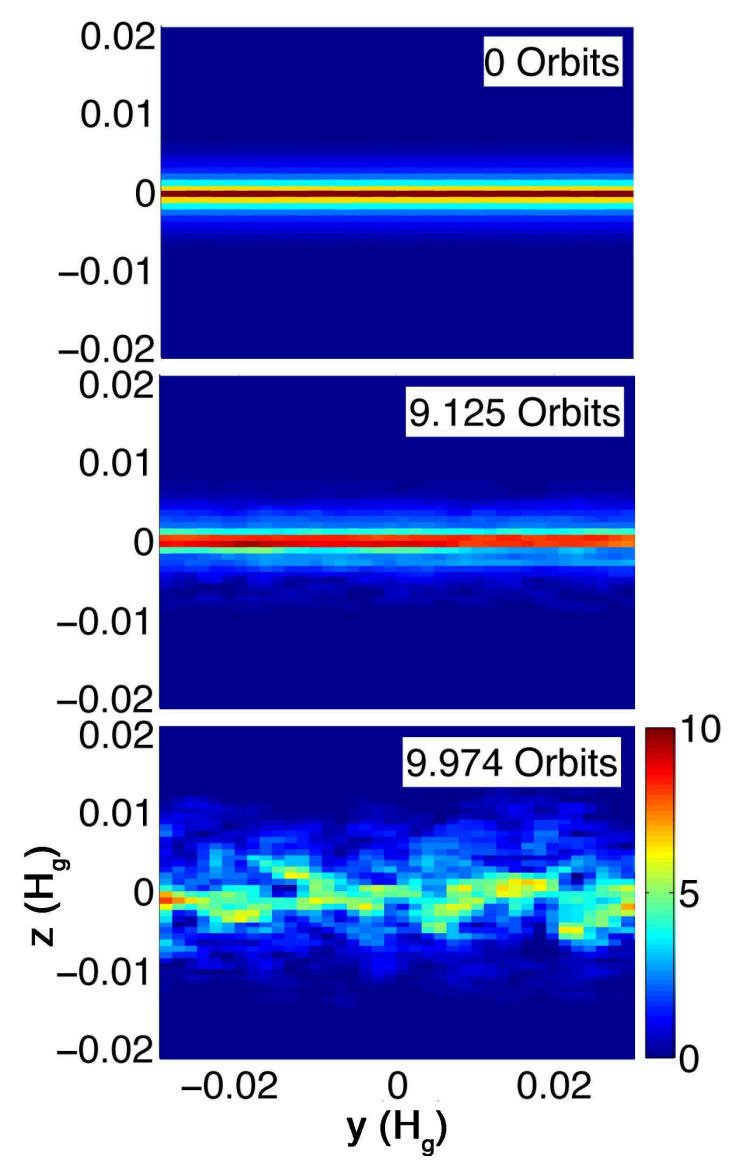

FIG. 4. - Snapshots of $\mu(y, z)$, sampled at $r=R(x=0$; the central slice of the simulation box) for the same unstable run shown in Figure 3 The box size parameters are $\left(L_{r}, L_{\phi}, L_{z}\right)=$ $(0.05,0.1,0.063) H_{\mathrm{g}}$, larger than what is shown in the figure, which zooms in for more detail.

seems correct only for dust-rich layers having $\mu_{0}$ so large they are on the verge of gravitational instability. For less dusty midplanes, the assumption appears to be incorrect. Our numerical results are roughly consistent with those of Chiang (2008), who also found evidence that $R i_{\text {crit }}$ decreases with decreasing $\mu_{0}$. Comparing his Table 2 with our Figure 5 shows that his constraints on $R i_{\text {crit }}$ are, for the most part, compatible with those presented here, for the range $\mu_{0} \approx 0.3-10$ where our respective data overlap. Our findings supersede those of Chiang (2008) insofar as we have explored parameter space more finely and systematically, at greater and more uniform resolution, with numerical methods better suited for subsonic flows.

Our results turn out to be consistent with the classical Richardson criterion - which states only that $R i<1 / 4$ is necessary, not sufficient, for instability - even though the criterion as derived by Miles (1961) applies only to twodimensional flows, which our dust layers are not. Our simulations demonstrate that the criterion can still serve as a useful guide for assessing stability in disks having bulk metallicities ranging from subsolar to slightly supersolar values - with the proviso that the actual Richardson number dividing $\mathrm{KH}$-stable from $\mathrm{KH}$-unstable flows, while $<1 / 4$, is generally not equal to $1 / 4$.

Why isn't the Richardson criterion for instability sufficient in rotating dust disks? The criterion considers the competition between the destabilizing vertical

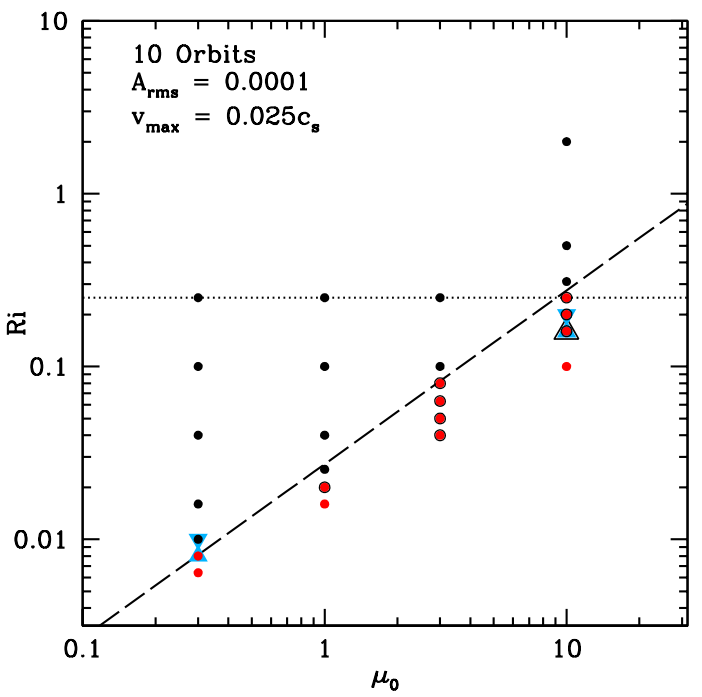

FIG. 5.- Mapping the boundary of stability in the space of initial $R i$ and $\mu_{0}$. Red points correspond to unstable dust layers, whose dust-to-gas ratios $\langle\mu\rangle$ change by more than $15 \%$, and whose vertical kinetic energies grow exponentially, within the 10-orbit duration of the simulation. Black points mark stable dust layers satisfying neither criterion. Red points outlined in black signify marginally unstable layers, whose kinetic energies rise but whose dust-to-gas ratios change by less than $15 \%$; these are essentially equivalent to red points without outlines, because every marginally unstable run that we extend beyond 10 orbits eventually becomes fully unstable. Runs performed at twice the standard resolution appear as triangles. Downward pointing triangles symbolize stable runs, upward triangles are unstable, and upward pointing triangles in black outline are marginally unstable. All simulations use $A_{\mathrm{rms}}=10^{-4}$ and $v_{\max }=0.025 c_{\mathrm{s}}$. There is no unique value for the critical Richardson number separating stable from unstable dust layers. Rather, a least-squares fit to the data from our standard resolution runs yields $R i_{\text {crit }} \propto \mu^{1.0}$, shown as a dashed line. The classical boundary $R i_{\text {crit }}=0.25$ is plotted as a dotted line.

shear and the stabilizing influence of buoyancy, which causes fluid parcels to oscillate about their equilibrium positions at the Brunt-Väisälä frequency. However, there exists another stabilizing influence, ignored by the Richardson number, provided by the radial Kepler shear (Ishitsu \& Sekiya 2003). In the limit $\mu_{0} \ll 1$, the Brunt frequency (4) becomes negligible relative to the Kepler shearing frequency (7), suggesting stability now depends on the competition between the destabilizing vertical shear and stabilizing radial Kepler shear. We expect the flow to be stable as long as the Kepler shear can wind up unstable eigenmodes to higher radial wavenumbers before their amplitudes grow large enough to trigger nonlinear effects. This suggests that we replace the Richardson number with a "shearing number," defined by analogy as the square of the ratio of the Kepler shearing frequency to the vertical shearing frequency:

$$
S h \equiv \frac{|\partial \Omega / \partial \ln r|^{2}}{\left(\partial v_{\phi} / \partial z\right)^{2}} \propto\left(\frac{\Delta z}{\Delta v_{\phi}}\right)^{2} \propto R i \frac{1+\mu_{0}}{\mu_{0}}
$$

where we have used (3) and (6). By assuming $S h$ is constant for marginally stable dust profiles, we arrive at the relation

$$
R i_{\text {crit }} \propto \mu_{0} \text { for } \mu_{0} \ll 1 .
$$

What is surprising is that this trend, although expected to hold only for $\mu_{0} \ll 1$, appears to hold approximately 


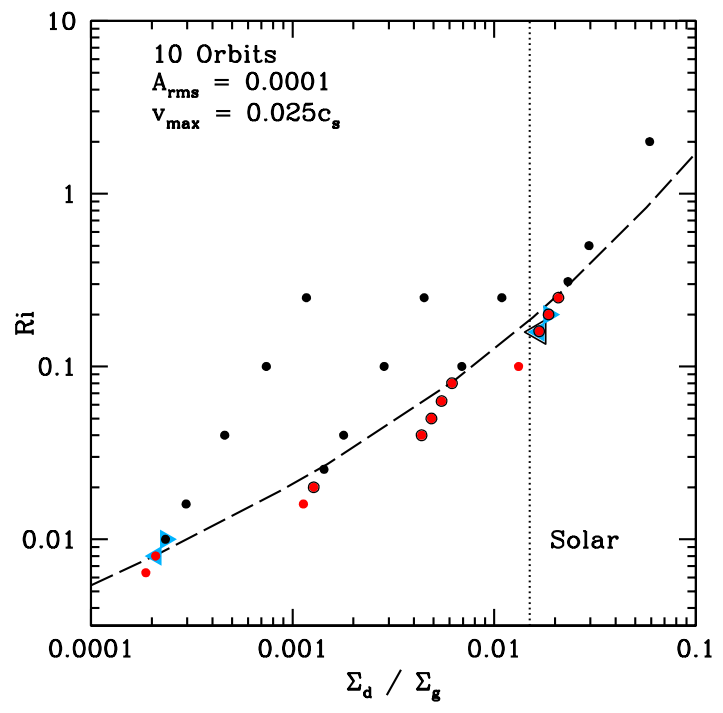

FIG. 6.- Mapping the boundary of stability in the space of initial $R i$ and bulk (height-integrated) dust-to-gas ratio $\Sigma_{\mathrm{d}} / \Sigma_{\mathrm{g}}$. The data are identical to those in Figure 5 . The labeling convention is also the same, except that the triangles representing high-resolution runs have adjusted their orientation so that they point towards the stability boundary. The same least-squares fit from Figure 5 is projected here as a dashed curve. Solar metallicity $\Sigma_{\mathrm{d}} / \Sigma_{\mathrm{g}}=0.015$ (Lodders 2003) is indicated by a dotted line. The critical value $R i_{\text {crit }}$ dividing stable from unstable dusty subdisks trends with metallicity. This trend was only hinted at in the data of C08.

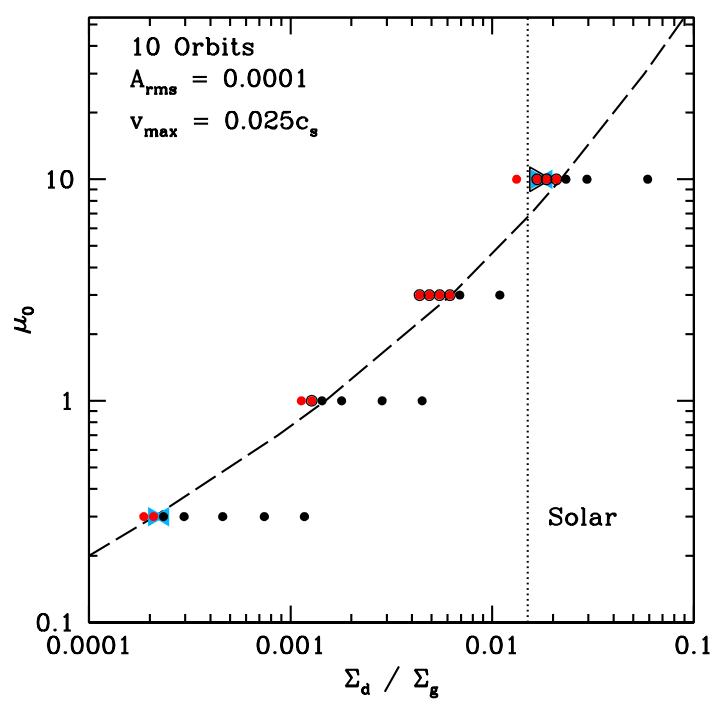

FIG. 7.- Mapping the boundary of stability in the space of midplane dust-to-gas ratio $\mu_{0}$ and bulk (height-integrated) dust-to-gas ratio $\Sigma_{\mathrm{d}} / \Sigma_{\mathrm{g}}$. The data are identical to those in Figure 5 The labeling convention is also the same, except that the triangles representing high-resolution runs have adjusted their orientation so that they point towards the stability boundary. The same least-squares fit from Figure 5 is projected here as a dashed curve. Solar metallicity $\Sigma_{\mathrm{d}} / \Sigma_{\mathrm{g}}=0.015$ (Lodders 2003) is indicated by a dotted line. A minimum-mass solar nebula requires $\mu_{0} \approx 30$ for gravitational instability to ensue on a dynamical time (CY10). Extrapolating the boundary of stability to $\mu_{0} \approx 30$ suggests that metallicities roughly $\sim 3$ times solar would be required for dynamical gravitational instability in a minimum-mass disk. The required degree of metal enrichment would be proportionately less in more massive disks.

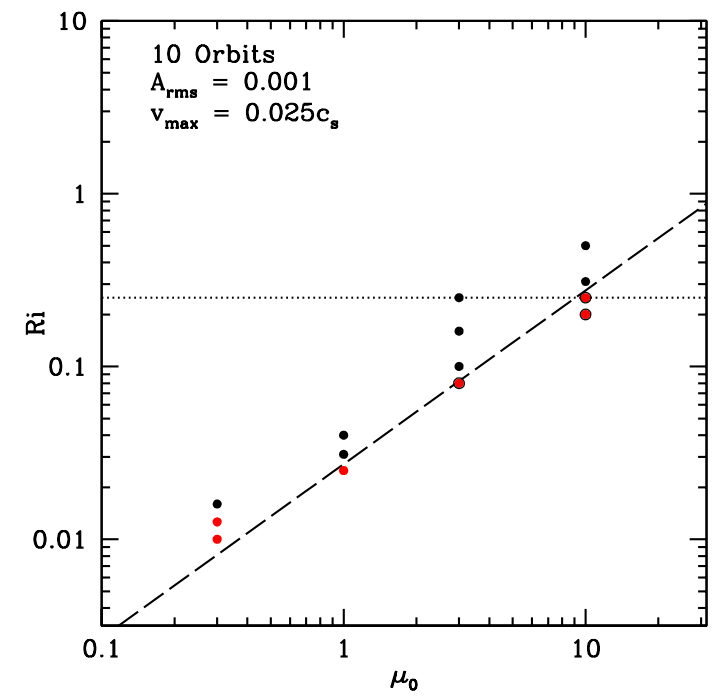

FIG. 8.- How the stability boundary changes with stronger initial perturbations. This figure is the same as Figure 5] except that all data correspond to $A_{\mathrm{rms}}=10^{-3}$. For comparison with $A_{\mathrm{rms}}=$ $10^{-4}$, the same best-fit line of Figure [5] is reproduced here. Not much changes, except that $R i_{\text {crit }}$ shifts upward by 0.2 dex at $\mu_{0}=$ 0.3 .

for all $\mu_{0}$, according to our simulation results in Figure 5 . For $\mu_{0} \gtrsim 1$, we would have expected from (32) that $R i_{\text {crit }}$ asymptote to a constant; but it does not. Our higher resolution runs do suggest the stability curve slightly flattens at $\mu_{0} \approx 10$, but such deviations seem too small to be fully explained using arguments relying purely on the shearing number.

To explain the observed trend, we might co-opt the methods of Ishitsu \& Sekiva (2003), who linearized and numerically integrated the 3D equations of motion for the dust layer. For their particular choice of background vertical density profile, they solved for the maximum growth factors for the most unstable KH modes (see also Knobloch \& Spruit 1985 who considered the axisymmetric problem). We would need to replace their assumed profile with our profiles having spatially constant $R i$. Perhaps our numerically determined stability curve $R i_{\text {crit }}\left(\Sigma_{\mathrm{d}} / \Sigma_{\mathrm{g}}\right)$ corresponds to a locus of fixed maximum growth factor.

Gravitational instability occurs on a dynamical time when the dust layer's Toomre $Q \approx M /\left[2 \pi r^{3} \rho_{\mathrm{g}}\left(1+\mu_{0}\right)\right]$ reaches unity (Toomre 1964; Goldreich \& Lynden-Bell 1965). For $\rho_{\mathrm{g}}$ given by the minimum-mass solar nebula, this occurs when $\mu_{0} \approx 30$, fairly independently of $r$ (Chiang \& Youdin 2010). Of course in more massive gas disks (greater $\rho_{\mathrm{g}}$ ), the requirement on $\mu_{0}$ is proportionately lower. Figure 7 shows that for disks having bulk metallicities $\Sigma_{\mathrm{d}} / \Sigma_{\mathrm{g}}$ equal to the solar value of 0.015 , the dusty sublayer can achieve $\mu_{0} \approx 8$ before it becomes KH unstable. Taken at face value, such a marginally $\mathrm{KH}$-stable subdisk, embedded in a gas disk having $30 / 8 \approx 4$ times the mass of the minimum-mass solar nebula, would undergo gravitational instability on the fastest timescale imaginable, the dynamical time. The case that planets form from disks several times more massive than the minimum-mass solar nebula is plausible (e.g., Goldreich et al. 2004; Lissauer et al. 2009).

An alternate way of crossing the Toomre threshold is 
to allow the bulk metallicity $\Sigma_{\mathrm{d}} / \Sigma_{\mathrm{g}}$ to increase above the solar value of 0.015 . Extrapolating the boundary of stability in Figure 7 to $\mu_{0} \approx 30$ suggests that metallicities roughly $\sim 3$ times solar would be required for dynamical gravitational instability in a minimum-mass disk. There are several proposed ways to achieve supersolar metallicities in some portions of the disk, among them radial pileups (Youdin \& Shu 2002) or dissipative gravitational instability (Ward 1976; Coradini et al. 1981; Ward 2000; Youdin 2005a; Youdin 2005b; see also the introduction of Goodman \& Pindor 2000).

None of the ways we have outlined for achieving gravitational instability rely on the streaming instability or turbulent concentration of particles, mechanisms that we have criticized in $\$ 1.3$. Nevertheless our scenarios may be too optimistic because all our dust profiles are predi- cated on the assumption of a spatially constant $R i$. This assumption tends to generate strong density cusps at the midplane that might not be present in reality. In a forthcoming paper we will relax the assumption of spatially constant $R i$ and measure the maximum $\mu_{0}$ attainable, as a function of metalllicity $\Sigma_{\mathrm{d}} / \Sigma_{\mathrm{g}}$, by simulating explicitly the settling of dust towards the midplane.

We thank Daniel Lecoanet, Eve Ostriker, Prateek Sharma, Jim Stone, and Yanqin Wu for discussions. An anonymous referee provided a thoughtful and encouraging report that helped to place our work in a broader context. This research was supported by the National Science Foundation, in part through TeraGrid resources provided by Purdue University under grant number TGAST090079.

\section{REFERENCES}

Bai, X. \& Goodman, J. 2009, ApJ, 701, 737

Balbus, S. A. 2009, ArXiv e-prints

Barranco, J. A. 2009, ApJ, 691, 907 (B09)

Barranco, J. A. \& Marcus, P. S. 2000, in Studying Turbulence Using Numerical Simulation Databases, 8. Proceedings of the 2000 Summer Program, p. 97, 97-+

Barranco, J. A. \& Marcus, P. S. 2005, ApJ, 623, 1157

-. 2006, Journal of Computational Physics, 219, 21

Blum, J. \& Wurm, G. 2008, ARA\&A, 46, 21

Chandrasekhar, S. 1981, Hydrodynamic And Hydromagnetic Stability, 1st edn. (Dover Publications, New York)

Chiang, E. 2008, ApJ, 675, 1549 (C08)

Chiang, E. \& Youdin, A. 2010, Annual Reviews of Earth and Planetary Science, 38 (CY10)

Coradini, A., Magni, G., \& Federico, C. 1981, A\&A, 98, 173

Cuzzi, J. N., Dobrovolskis, A. R., \& Champney, J. M. 1993, Icarus, 106, 102

Cuzzi, J. N., Hogan, R. C., \& Shariff, K. 2008, ApJ, 687, 1432

Drazin, P. G. \& Reid, W. H. 2004, Hydrodynamic Stability, 2nd edn. (Cambridge University Press, Cambridge)

Eaton, J. K. \& Fessler, J. R. 1994, International Journal of Multiphase Flow Supplemental, 20, 169

Gammie, C. F. 1996, ApJ, 462, 725

Gilman, P. A. \& Glatzmaier, G. A. 1981, ApJS, 45, 335

Goldreich, P., Lithwick, Y., \& Sari, R. 2004, ARA\&A, 42, 549

Goldreich, P. \& Lynden-Bell, D. 1965, MNRAS, 130, 125

Goldreich, P. \& Ward, W. R. 1973, ApJ, 183, 1051

Gómez, G. C. \& Ostriker, E. C. 2005, ApJ, 630

Goodman, J. \& Pindor, B. 2000, Icarus, 148, 537

Gough, D. O. 1969, Journal of Atmospheric Sciences, 26, 448

Hogan, R. C. \& Cuzzi, J. N. 2007, Phys. Rev. E, 75, 056305

Ilgner, M. \& Nelson, R. P. 2006, A\&A, 445, 223

Ishitsu, N. \& Sekiya, M. 2003, Icarus, 165, 181

Johansen, A., Henning, T., \& Klahr, H. 2006, ApJ, 643, 1219
Johansen, A., Oishi, J. S., Low, M., Klahr, H., Henning, T., \& Youdin, A. 2007, Nature, 448, 1022

Johansen, A., Youdin, A., \& Mac Low, M. 2009, ApJ, 704, L75

Knobloch, E. \& Spruit, H. C. 1985, Geophysical and

Astrophysical Fluid Dynamics, 32, 197

-. 1986, A\&A, 166, 359

Lecoanet, D., Zweibel, E. G., Townsend, R. H. D., \& Huang, Y. 2010, ApJ, 712, 1116

Lissauer, J. J., Hubickyj, O., D’Angelo, G., \& Bodenheimer, P. 2009, Icarus, 199, 338

Lithwick, Y. 2009, ApJ, 693, 85

Lodders, K. 2003, ApJ, 591, 1220

Maxey, M. R. 1987, J. Fluid Mech., 174, 441

Miles, J. W. 1961, Journal of Fluid Mechanics, 10, 496

Nakagawa, Y., Sekiya, M., \& Hayashi, C. 1986, Icarus, 67, 375

Ogura, Y. \& Phillips, N. A. 1962, Journal of Atmospheric Sciences, 19

Safronov, V. S. 1969, Evolution of the protoplanetary cloud and formation of the Earth and planets (IPST Jerusalem)

Sekiya, M. 1998, Icarus, 133, 298

Toomre, A. 1964, ApJ, 139, 1217

Turner, N. J., Carballido, A., \& Sano, T. 2010, ApJ, 708, 188

Ward, W. R. 1976, in Frontiers of Astrophysics, 1-40

Ward, W. R. On Planetesimal Formation: The Role of Collective Particle Behavior, ed. Canup, R. M., Righter, K., \& et al., $75-84$

Weidenschilling, S. J. 1980, Icarus, 44, 172

Wilner, D. J., D'Alessio, P., Calvet, N., Claussen, M. J., \& Hartmann, L. 2005, ApJ, 626, L109

Youdin, A. N. 2005a, ArXiv Astrophysics e-prints -. 2005b, ArXiv Astrophysics e-prints

Youdin, A. N. \& Chiang, E. I. 2004, ApJ, 601, 1109

Youdin, A. N. \& Goodman, J. 2005, ApJ, 620, 459

Youdin, A. N. \& Shu, F. H. 2002, ApJ, 580, 494 